\title{
Transforming growth factor $\beta$ and its role in heart disease (Review)
}

\author{
GUANGWANG LIU ${ }^{1,2}$, CHAO MA ${ }^{2}$, HUILIN YANG ${ }^{1}$ and PEI-YING ZHANG ${ }^{2}$ \\ ${ }^{1}$ Institute of Orthopedics, Soochow University, Soochow, Jiangsu 215006; ${ }^{2}$ Department of Cardiology, \\ Xuzhou Central Hospital, The Affiliated Xuzhou Hospital of Medical College \\ of Southeast University, Xuzhou, Jiangsu 221009, P.R. China
}

Received June 16, 2016; Accepted December 27, 2016

DOI: $10.3892 /$ etm.2017.4246

\begin{abstract}
Myocardial infarction (MI) is a major form of heart disease that leads to immediate cardiomyocyte death due to ischemia and eventually fibrosis and scar formation and further dysfunction of myocardium and heart failure. Extracellular matrix (ECM) production and tissue repair is conducted by myofibroblasts, which are formed from the normal quiescent cardiac fibroblasts following transformational changes, through the active participation of transforming growth factor $\beta$ (TGF $\beta$ ) and its signaling pathways. TGF $\beta$ appears to be a 'Master of all trades', with respect to cardiac fibrosis, as it can promote cardiomyocyte apoptosis and cardiac hypertrophy. TGF $\beta$ signaling involves its binding to TGF $\beta$ receptor type II (TGF $\beta$ RII), which recruits TGF $\beta$ receptor type I (TGF $\beta$ RI), which are also known as activin receptor-like kinase (ALK) in five different isoforms. In canonical signaling pathways, ALK5 activates Smads 2 and 3, and ALK1 activates Smads 1 and 5. These pairs of Smads form a corresponding complex and then bind to Smad 4, to translocate into the nucleus, where transcriptional reprogramming is carried out to promote myofibroblast formation and ECM production, eventually leading to cardiac fibrosis. TGF $\beta$ levels are elevated in MI, thereby aggravating the myocardial injury further. Several microRNAs are involved in the regulation of TGF $\beta$ signaling at different steps, affecting different components. Therapeutic
\end{abstract}

Correspondence to: Professor Huilin Yang, Institute of Orthopedics, Soochow University, Soochow, Jiangsu 215006, P.R. China

E-mail: hlyang@suda.edu.cn

Professor Pei-Ying Zhang, Department of Cardiology, Xuzhou Central Hospital, The Affiliated Xuzhou Hospital of Medical College of Southeast University, 199 South Jiefang Road, Xuzhou, Jiangsu 221009, P.R. China

E-mail: zpying58@126.com

Key words: transforming growth factor $\beta$, activin receptor-like kinase, Smads, extracellular matrix, cardiac fibrosis, apoptosis, myofibroblasts targeting of TGF $\beta$ signaling at ALK1-5 receptor activity level has met with limited success and extensive research is needed to develop therapies based on the components of TGF $\beta$ signaling pathway, for instance cardiac dysfunction and heart failure.

\section{Contents}

1. Introduction

2. TGF $\beta$ and cardiac fibroblast transformation

3. TGF $\beta$ receptors and signaling

4. TGF $\beta$ signaling and miRNAs

5. Pathological effects of TGF $\beta$ and Smad signaling

6. Conclusions

\section{Introduction}

Heart diseases affect millions of individuals worldwide and the mortality due to different types of heart diseases, both congenital and acquired is steadily increasing. Myocardial infarction (MI) is a major form of heart disease that leads to immediate cardiomyocyte death due to ischemia. Even intervention with reperfusion in a timely manner, causes reperfusion injury to the myocardium, by promoting post-infarct heart remodeling, which is a known cause of heart failure (1).

A fundamental problem during heart remodeling is cardiac fibrosis, which is promoted by the formation of myofibroblasts and excessive section of extracellular matrix (ECM). There are three major types of cells in the heart, including cardiomyocytes, which are contractile, fibroblasts, which consist of $10-30 \%$ of total cells, i.e., even more than cardiomyocytes (1), in adult heart and provide structural support and vascular cells, which are important for vascularization (2). Fibroblasts are known to secrete ECM into the interstitial space, which is necessary for providing structural organization and support to myocardium $(3,4)$ and thus play an important role in tissue replacement and repair following injury to myocardium. However, this ability of ECM production and tissue repair is bestowed upon the normal quiescent cardiac fibroblasts following transformational changes into myofibroblasts, accompanied by several changes in gene expression and 
phenotype of the cells. Hypertension and many other vascular and heart diseases lead to cardiac fibrosis, which compromises the mechanical function of heart and thus pose serious threat to overall health and survival $(3,4)$. Previous findings suggested that conditions associated with elevated levels of plasma levels of certain hormones such as aldosterone, angiotensin II, endothelin-1 (ET-1) and cytokines including transforming growth factor $\beta$ (TGF $\beta)$, connective tissue growth factor $(\mathrm{CTGF} / \mathrm{CCN} 2)$ and platelet-derived growth factor (PDGF), which actively participate in the process of transformation of quiescent fibroblasts to myofibroblasts, lead to cardiac fibrosis and eventually to heart failure (5). Normally, myofibroblasts undergo apoptotic removal following their function of tissue repair, but their persistent presence in disease and stress conditions, results in excessive ECM production and cardiac remodeling and fibrosis (6). Fibrotic response arises from a concerted action of hormones such as aldosterone, ET-1 and angiotensin II and cytokines TGF $\beta$, with the matricellular CTGF/CCN2, which amplifies the signals coming from TGF $\beta$ (7).

In this review, we discuss the role of TGF $\beta$ and its signaling in cardiac fibrosis and remodeling and heart failure. TGF $\beta$ plays an important role in post-infarct remodeling, where tissue repair involving hypertrophic growth and fibrosis take place as a compensatory mechanism in response to the loss of cardiomyocytes by apoptosis (8).

\section{TGF $\beta$ and cardiac fibroblast transformation}

It is well established that TGF $\beta$ expression is increased in response to tissue injury in general (9) and this cytokine is involved in the tissue repair process and scar formation (10). Notably, TGF $\beta$ appears to be a 'Master of all trades', with respect to cardiac fibrosis (11), as it can promote cardiomyocyte apoptosis (12) and cardiac hypertrophy (13). Additionally, TGF $\beta$ is upregulated in post-infarction myocardium (14) and this correlates strongly with reduced ventricular ejection fractions (15). There are three TGF $\beta$ isoforms, TGF $\beta 1$, TGF $\beta 2$ and TGF $\beta 3$ and all these are released from their binding proteins, present as a protein complex, via the proteolytic process. Although most cells in myocardium are known to release TGF $\beta 1$, macrophages that infiltrate into myocardium after myocardial injury and cardiomyocyte apoptosis to engulf the damaged cardiomyocytes, are known to release TGF $\beta$ and angiotensin II in significant quantities (16). It has been demonstrated that TGF $\beta$, when added to fibroblast cultures in vitro, induces the expression of genes related to ECM production and thus increases ECM deposition and concomitant suppression of matrix metalloproteinase through elevation of inhibitors of matrix metalloproteinase gene expression (7). TGF $\beta$ binds to its type I and II receptors and TGF $/ \mathrm{Smad}$ signaling (see below) in fibroblasts, which sets into motion the processes involved in the transformation of fibroblasts to myofibroblasts. Once formed, myofibroblasts not only secrete ECM components, but also TGF $\beta$, angiotensin and ET-1, which in a cyclical manner, further increase the formation of more fibroblasts and ECM deposition (16). The role of TGF $\beta$ in scar formation is evident from studies showing that the treatment of wounds with anti-TGF $\beta$-antibodies or antisense oligonucleotides directed against TGF $\beta$, reduce both ECM production as well as scarring (17). MI and ischemia reperfusion injury to myocardium are known to elevate the levels of reactive oxygen species (ROS) in myocardial cells and this is known to increase the expression of TGF $\beta$, which further aggravates injury and in fact the treatment of MI patients with ROS scavenger, $\mathrm{N}$-acetylcysteine, was found to reduce TGF $\beta$ levels (15). Besides the transformation of fibroblasts, TGF $\beta 1$ by partnering with tumor necrosis factor- $\alpha$ or IL1- $\beta$, is also shown to promote epithelial to mesenchymal transdifferentiation and endothelial to mesenchymal transdifferentiation, both of which contribute to the formation of myofibroblasts $(18,19)$. It is estimated that nearly $35 \%$ of the fibroblasts in the fibrotic areas of heart are derived through the process of endothelial to mesenchymal transdifferentiation (20).

\section{TGF $\beta$ receptors and signaling}

TGF $\beta$ exerts its cellular effects through its binding to cell surface receptors, TGF $\beta$ receptors type I (TGF $\beta$ RI) and type II (TGFßRII), which are Ser/Thr kinase receptors. Engagement of TGF $\beta 1$ to TGF $\beta R I I$ induces its autophosphorylation, resulting in the recruitment of TGF $\beta$ RI, which is also known as activin receptor-like kinase (ALK), and its heterodimerization with TGF $\beta R I$ (21). After recruitment, TGF $\beta$ RI phosphorylates and activates receptor-mediated Smads (R-Smad2 and R-Smad3), the downstream players in the TGF $\beta$ signaling pathway (22). R-Smads are released from Smad anchor after phosphorylation by ALK in response to TGF $\beta$, and then they form a complex with co-mediator Smad4 (co-Smad4). This Smad complex translocates into the nucleus (Fig. 1), where it binds to promoter regions of the genes involved in ECM production and fibrotic process and enhances their expression. TGF $\beta$ signaling gets more modular and cell specific as there are five different isoforms of TGF $\beta$ RI (ALK1-5), which are expressed in different cell types and function by activating Smads 2 and 3 or Smads 1 and 5 (23). ALK1 mediates the activation of Smads 1/5 whereas ALK5 activates Smads 2/3 in endothelial cells and in many other cell types, thus adding another layer of complexity to TGF $\beta$ signaling. In addition, Smads 6 and 7 are known as inhibitory Smads that counter-regulate TGF $\beta$ signaling (Fig. 1). Thus, the outcome of TGF $\beta 1$ signaling is a balance between stimulatory R-Smad $2 / 3$ and inhibitory Smad6/7 (I-Smad6/7). In addition to Smads 6 and 7, other factors such as Ski, and SnoN also act as negative regulators of TGF $\beta$ signaling $(24,25)$. I-Smad7, which itself is induced by TGF $\beta$, acts as a negative feedback regulator by targeting TGF $\beta R$ I for degradation through Smurf2, a Smad ubiquitination protein (26). By contrast, SnoN and Ski, which are ubiquitously expressed, negatively modulate TGF- $\beta 1$ signaling at the gene transcription. Besides ALK-mediated signaling, TGF $\beta$ also mediates signaling directly via TGF $\beta$ RII, by activating kinases such as TAK1, RhoA, ERK, and p38 (27) and also through the regulation of microRNAs (miRNAs), both at the transcriptional and post-transcriptional level (28).

Importance of Smad 3 in TGF $\beta$-mediated signaling and subsequent fibrotic response became evident in studies using Smad 3-knockout (KO) mice. Thus, it has been demonstrated that there is accelerated wound healing and low scar tissue formation, and reduced inflammation in Smad3-KO mice (29). Moreover, infarcted hearts from Smad3-KO mice display 


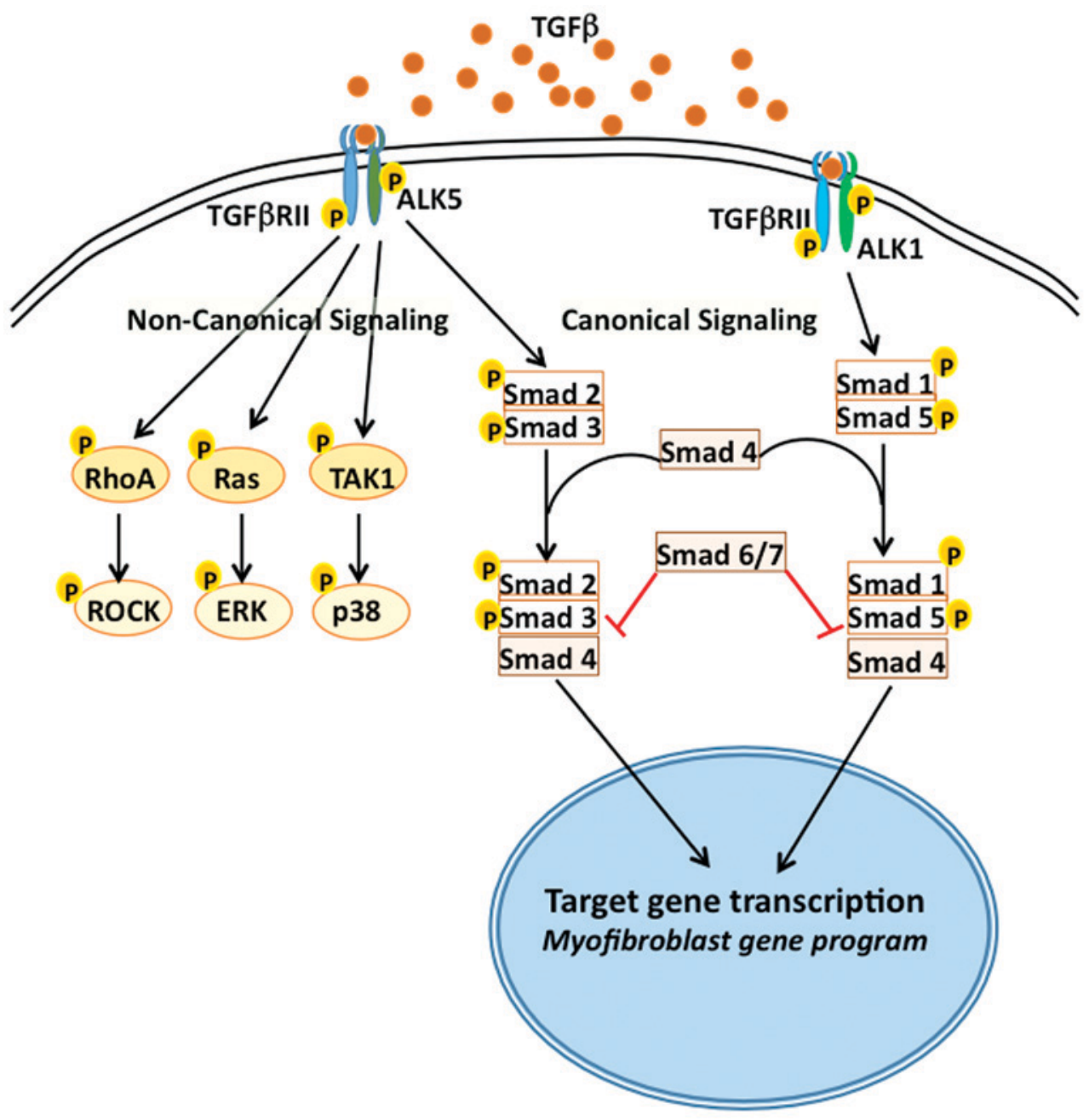

Figure 1. TGF $\beta$ signaling pathways that lead to myofibroblast formation. Binding of TGF $\beta$ to TGF $\beta$ RII leads to its autophosphorylation and recruitment of TGFßRI (also known as ALK1-5). In normal canonical signaling, Smads 2 and 3 are activated by ALK5, followed by complex formation with Smad 4, for translocation into nucleus and activation of transcriptional program relevant for myofibroblast transformation. ALK1 activates Smads 1 and 5. Translocation of Smad complex into nucleus is inhibited by Smads 6 and 7, which prevent myofibroblast formation. In non-canonical signaling of TGF $\beta 1$, TGF $\beta R$ II phosphorylates and activates RhoA, Ras and/or TAK1, which further activate ROCK, ERK and p38, respectively. TGF $\beta$, transforming growth factor $\beta$; TGF $\beta$ RII, transforming growth factor receptor type II; TGF $\beta$ R, transforming growth factor receptor type I; ALK, activin receptor-like kinase.

reduced cardiac fibrosis in comparison to wild-type mice (30). Isolated cardiac fibroblasts from Smad3-KO mice are unable to respond to TGF $\beta$ to produce elevated levels of collagen and other ECM components, such as pro-collagen III and tenascin-C $(31,32)$, indicating the essential role of Smad 3 in TGF $\beta$ signaling and fibrotic processes. Non-canonical pathways of TGF $\beta$ signaling are also involved in the expression of $\alpha$-smooth muscle actin ( $\alpha$ SMA) expression and CTGF/CCN2 expression by the myofibroblasts $(33,34)$.

\section{TGF $\beta$ signaling and miRNAs}

miRNAs are important in the modulation of TGF $\beta$ signaling and cardiac fibrosis. Thus, it has been shown earlier that miR24 prevents conversion of latent TGF $\beta$ to active form (35) and protects myocardium from post-infarction apoptosis and loss of cardiomyocytes in transgenic mice with cardiomyocyte-specific overexpression of miR24 (36). Similarly, it has been observed that miR503 is upregulated in the mouse left ventricles subjected to transverse aortic constriction and also in neonatal cardiac fibroblasts treated with angiotensin II in cell culture. miR-503 has been found to promote cardiac fibrosis involving the Apelin-13-TGF $\beta$-CTGF-collagen production pathway (37). In another study, miR101a was shown to protect from hypoxia-induced cardiac fibrosis by targeting TGF $\beta$ RI (ALK) in cardiac fibroblasts (38). Another miRNA, miR92a appears to be involved in promoting fibrosis induction by TGF $\beta$ by maintaining inhibitory Smad 7 at low levels. Thus, antagomirs against miR92a have been shown to protect hypoxia/reoxygenation induced apoptosis of cardiomyocytes (39). In a more recent study, it has been shown that miR19a-3p/19b-3p was present at low levels in the plasma of heart failure patients and that miR19a-3p/19b-3p mimics are inhibitory to epithelial mesenchymal transition and ECM production and invasion of cardiac fibroblasts. These miRs are found to antagonize autophagy of cardiac fibroblasts by targeting TGF $\beta$ RII mRNA (40). In an elegant study, Tijsen et al (41) demonstrated that expression of the miR15 family was elevated in models of overloaded heart and cardiac hypertrophy and fibrosis in a protective response, as antimiRs against miR15 aggravated the fibrosis and hypertrophy in mice. miR15 was found to target TGF $\beta$ RI (ALK) and other 


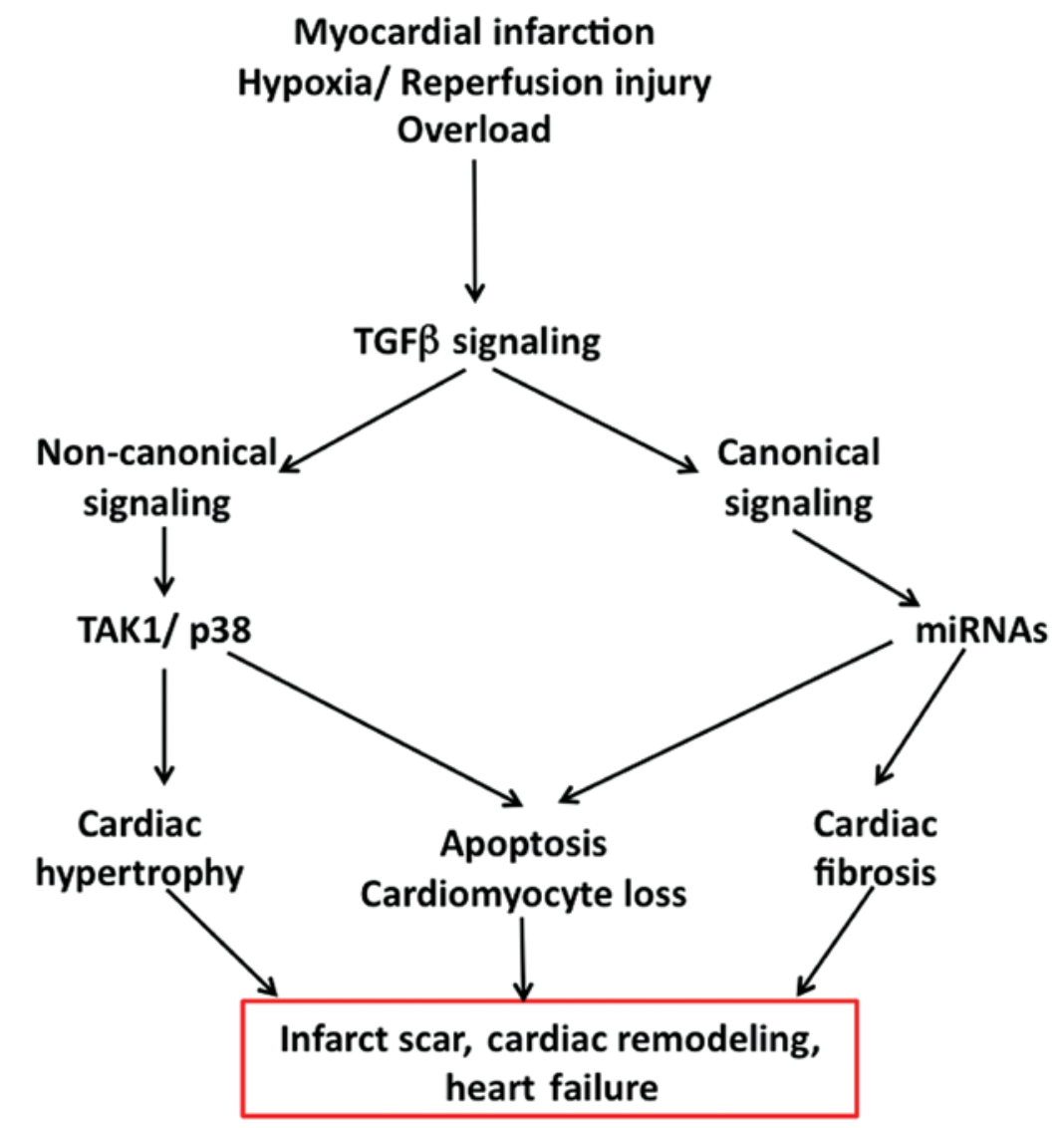

Figure 2. Pathological effects of TGF $\beta$ signaling and heart failure. Hypoxia/reperfusion injury, overload and/or myocardial infarction lead to heart dysfunction through TGF $\beta$ signaling. The canonical and non-canonical signaling pathways of TGF $\beta$ trigger cardiac hypertrophy, apoptosis of cardiomyocytes and fibrosis, which all culminate in scar formation in the infarct area, cardiac remodeling and eventually heart failure. TGF $\beta$, transforming growth factor $\beta$.

components of this signaling pathway. Thus it appears that several miRNAs are involved in the regulation of TGF $\beta$ signaling and its effects on cardiac function.

\section{Pathological effects of TGF $\beta$ and Smad signaling}

As mentioned before, several lines of experimental evidence suggest the importance of Smad signaling in TGF $\beta$-mediated detrimental effects in cardiac dysfunction and failure (Fig. 2). Thus, it has been shown that decoy-oligonucleotides targeting Smads were found to prevent TGF $\beta$-induced apoptosis in cardiomyocytes (12). Interruption of Smad 2 signaling by inhibiting ALK receptors using SB431542, also prevented TGF $\beta$-induced apoptosis in cardiomyocytes (42). As detailed above, targeting Smads with miRNAs has been shown to be protective against TGF $\beta$-mediated detrimental effects (36). Of note, it has been demonstrated that activation of alternate Smad signaling pathways is able to prevent MI-mediated cardiomyocyte death. Thus, bone morphogenetic protein-2 (BMP2), another TGF family member, which activates Smad 1/5/8 signaling via ALK1, 2 or 3 receptors, is able to alleviate post-MI cardiomyocyte death and improve heart function (43). Additionally, endothelial mesenchymal transdifferentiation, which is known to contribute to myofibroblasts, is significantly decreased in Smad 3-KO mice and also by injection of BMP7 (44). Smad 3-KO mice have been found to display reduced fibrosis following MI. Protected diastolic function and isolated cardiac fibroblasts from these $\mathrm{KO}$ mice do not show enhanced collagen synthesis in response to TGF $\beta$ and also markedly lowered migration and transdifferentiation potential to become myofibroblasts $(30,45)$. Inasmuch as Smad 3-KO did not alter early immune and inflammatory responses of myocardium, Smad 3 pathway is a potential therapeutic target for reduction of the fibrotic response following MI and hypoxia/reperfusion injury. There is gene dosage effect on TGF $\beta$ signaling with regard to Smad 3 expression, as mice heterozygous for Smad3 are protected from cardiac hypertrophy induced by diabetes (46). Novel ALK inhibitors such as GW788388 have been found to curtail Smad 2 activation, myofibroblast formation, ECM deposition as well as systolic dysfunction, without changing TGF $\beta$ levels and macrophage infiltration, which is necessary for myocardial injury healing (47). However, other ALK inhibitors were found to have several unwanted side effects including increased mortality and valve lesions $(48,49)$, thus raising concerns in targeting this target. Overexpression of inhibitory Smad 7 in vivo, inhibited angiotensin II-induced fibrosis and loss of contractility, whereas in vitro overexpression curtailed ROS-induced expression of matrix metalloproteases and collagen (50).

Besides the canonical pathways, non-canonical signaling through TAK1 is important in TGF $\beta$-mediated effects on cardiac dysfunction and hypertrophic response. TAK1-mediated effects appear to involve p38 MAPK and other effectors such as TAK1 binding protein and JNK kinases (51). Extensive understanding of this complex signaling pathway is 
imperative in order to develop therapies targeting the components of TGF $\beta$ signaling pathway.

\section{Conclusions}

MI leads to immediate cardiomyocyte death due to ischemia and fibrosis and further dysfunction of myocardium and heart failure. Fibrosis is deposition of ECM, which is conducted by myofibroblasts, which are formed from the normal quiescent cardiac fibroblasts, through the active participation of TGF $\beta$ and its signaling pathways. TGF $\beta$ appears to be responsible for cardiac fibrosis, cardiomyocyte apoptosis and cardiac hypertrophy. TGF $\beta$ signaling involves its binding to TGF $\beta$ RII receptor, which recruits TGF $\beta$ RI receptors, also known as ALK in five different isoforms. Canonical signaling pathways of TGF $\beta$ involve activation of Smads, which translocate into nucleus, where transcriptional reprogramming is carried out to promote myofibroblast formation and ECM production, eventually leading to cardiac fibrosis. TGF $\beta$ levels are elevated in $\mathrm{MI}$, thereby aggravating the myocardial injury further. Therapeutic targeting of TGF $\beta$ signaling at ALK1-5 receptor activity level has met with limited success and investigation is needed to develop therapies based on TGF $\beta$ signaling pathway, for cardiac dysfunction and heart failure.

\section{References}

1. Nag AC: Study of non-muscle cells of the adult mammalian heart: a fine structural analysis and distribution. Cytobios 28: 41-61, 1980.

2. Pinto AR, Ilinykh A, Ivey MJ, Kuwabara JT, D'Antoni ML, Debuque R, Chandran A, Wang L, Arora K, Rosenthal NA, et al: Revisiting cardiac cellular composition. Circ Res 118: 400-409, 2016

3. Souders CA, Bowers SL and Baudino TA: Cardiac fibroblast: the renaissance cell. Circ Res 105: 1164-1176, 2009.

4. Moore-Morris T, Cattaneo P, Puceat M and Evans SM: Origins of cardiac fibroblasts. J Mol Cell Cardiol 91: 1-5, 2016.

5. Hinz B, Phan SH, Thannickal VJ, Galli A, Bochaton-Piallat ML and Gabbiani G: The myofibroblast: one function, multiple origins. Am J Pathol 170: 1807-1816, 2007.

6. Leask A: Potential therapeutic targets for cardiac fibrosis: TGFbeta, angiotensin, endothelin, CCN2, and PDGF, partners in fibroblast activation. Circ Res 106: 1675-1680, 2010.

7. Leask A: Targeting the TGFbeta, endothelin- 1 and $\mathrm{CCN} 2$ axis to combat fibrosis in scleroderma. Cell Signal 20: 1409-1414, 2008.

8. Euler G: Good and bad sides of TGF $\beta$-signaling in myocardial infarction. Front Physiol 6: 66, 2015.

9. Kane CJ, Hebda PA, Mansbridge JN and Hanawalt PC: Direct evidence for spatial and temporal regulation of transforming growth factor beta 1 expression during cutaneous wound healing. J Cell Physiol 148: 157-173, 1991.

10. Massagué J: TGF-beta signal transduction. Annu Rev Biochem 67: 753-791, 1998.

11. Edgley AJ, Krum H and Kelly DJ: Targeting fibrosis for the treatment of heart failure: a role for transforming growth factor- $\beta$. Cardiovasc Ther 30: e30-e40, 2012.

12. Schneiders D, Heger J, Best P, Michael Piper H and Taimor G: SMAD proteins are involved in apoptosis induction in ventricular cardiomyocytes. Cardiovasc Res 67: 87-96, 2005.

13. Huntgeburth M, Tiemann K, Shahverdyan R, Schlüter KD, Schreckenberg R, Gross ML, Mödersheim S, Caglayan E, Müller-Ehmsen J, Ghanem A, et al: Transforming growth factor $\beta_{1}$ oppositely regulates the hypertrophic and contractile response to $\beta$-adrenergic stimulation in the heart. PLoS One 6: e26628, 2011.

14. Vilahur G, Juan-Babot O, Peña E, Oñate B, Casaní L and Badimon L: Molecular and cellular mechanisms involved in cardiac remodeling after acute myocardial infarction. J Mol Cell Cardiol 50: 522-533, 2011.
15. Talasaz AH, Khalili H, Jenab Y, Salarifar M, Broumand MA and Darabi F: N-Acetylcysteine effects on transforming growth factor- $\beta$ and tumor necrosis factor- $\alpha$ serum levels as pro-fibrotic and inflammatory biomarkers in patients following ST-segment elevation myocardial infarction. Drugs R D 13: 199-205, 2013.

16. Shinde AV and Frangogiannis NG: Fibroblasts in myocardial infarction: a role in inflammation and repair. J Mol Cell Cardiol 70: 74-82, 2014

17. Cordeiro MF, Mead A, Ali RR, Alexander RA, Murray S, Chen C, York-Defalco C, Dean NM, Schultz GS and Khaw PT: Novel antisense oligonucleotides targeting TGF-beta inhibit in vivo scarring and improve surgical outcome. Gene Ther 10: 59-71, 2003.

18. Yamauchi Y, Kohyama T, Takizawa H, Kamitani S, Desaki M, Takami K, Kawasaki S, Kato J and Nagase T: Tumor necrosis factor-alpha enhances both epithelial-mesenchymal transition and cell contraction induced in A549 human alveolar epithelial cells by transforming growth factor-beta1. Exp Lung Res 36: 12-24, 2010.

19. Maleszewska M, Moonen JR, Huijkman N, van de Sluis B, Krenning $G$ and Harmsen MC: IL- $1 \beta$ and TGF $\beta 2$ synergistically induce endothelial to mesenchymal transition in an NFאB-dependent manner. Immunobiology 218: 443-454, 2013.

20. Zeisberg EM, Tarnavski O, Zeisberg M, Dorfman AL, McMullen JR, Gustafsson E, Chandraker A, Yuan X, Pu WT, Roberts AB, et al: Endothelial-to-mesenchymal transition contributes to cardiac fibrosis. Nat Med 13: 952-961, 2007.

21. Wrana JL, Attisano L, Wieser R, Ventura F and Massagué J: Mechanism of activation of the TGF-beta receptor. Nature 370: 341-347, 1994.

22. Zhang Y, Feng X, We R and Derynck R: Receptor-associated Mad homologues synergize as effectors of the TGF-beta response. Nature 383: 168-172, 1996.

23. Wharton K and Derynck R: TGFbeta family signaling: novel insights in development and disease. Development 136: 3691-3697, 2009.

24. Cunnington RH, Nazari M and Dixon IM: c-Ski, Smurf2, and Arkadia as regulators of TGF-beta signaling: new targets for managing myofibroblast function and cardiac fibrosis. Can J Physiol Pharmacol 87: 764-772, 2009.

25. Wrana JL: Regulation of Smad activity. Cell 100: 189-192, 2000

26. Whitman M: Signal transduction. Feedback from inhibitory SMADs. Nature 389: 549-551, 1997.

27. Dobaczewski M, Chen W and Frangogiannis NG: Transforming growth factor (TGF) $-\beta$ signaling in cardiac remodeling. J Mol Cell Cardiol 51: 600-606, 2011.

28. Blahna MT and Hata A: Smad-mediated regulation of microRNA biosynthesis. FEBS Lett 586: 1906-1912, 2012.

29. Flanders KC, Sullivan CD, Fujii M, Sowers A, Anzano MA, Arabshahi A, Major C, Deng C, Russo A, Mitchell JB, et al: Mice lacking Smad 3 are protected against cutaneous injury induced by ionizing radiation. Am J Pathol 160: 1057-1068, 2002.

30. Bujak M, Ren G, Kweon HJ, Dobaczewski M, Reddy A, Taffet G, Wang XF and Frangogiannis NG: Essential role of Smad3 in infarct healing and in the pathogenesis of cardiac remodeling. Circulation 116: 2127-2138, 2007.

31. Yang YC, Piek E, Zavadil J, Liang D, Xie D, Heyer J, Pavlidis P, Kucherlapati R, Roberts AB and Böttinger EP: Hierarchical model of gene regulation by transforming growth factor beta. Proc Natl Acad Sci USA 100: 10269-10274, 2003.

32. Verrecchia F, Chu ML and Mauviel A: Identification of novel TGF-beta/Smad gene targets in dermal fibroblasts using a combined cDNA microarray/promoter transactivation approach. J Biol Chem 276: 17058-17062, 2001.

33. Santander C and Brandan E: Betaglycan induces TGF-beta signaling in a ligand-independent manner, through activation of the p38 pathway. Cell Signal 18: 1482-1491, 2006.

34. Shi-wen X, Parapuram SK, Pala D, Chen Y, Carter DE, Eastwood M, Denton CP, Abraham DJ and Leask A: Requirement of transforming growth factor beta-activated kinase 1 for transforming growth factor beta-induced alpha-smooth muscle actin expression and extracellular matrix contraction in fibroblasts. Arthritis Rheum 60: 234-241, 2009.

35. Wang J, Huang W, Xu R, Nie Y, Cao X, Meng J, Xu X, Hu S and Zheng Z: MicroRNA-24 regulates cardiac fibrosis after myocardial infarction. J Cell Mol Med 16: 2150-2160, 2012.

36. Guo C, Deng Y, Liu J and Qian L: Cardiomyocyte-specific role of miR-24 in promoting cell survival. J Cell Mol Med 19: 103-112, 2015. 
37. Zhou Y, Deng L, Zhao D, Chen L, Yao Z, Guo X, Liu X, Lv L, Leng B, Xu W, et al: MicroRNA-503 promotes angiotensin II-induced cardiac fibrosis by targeting Apelin-13. J Cell Mol Med 20: 495-505, 2016.

38. Zhao X, Wang K, Liao Y, Zeng Q, Li Y, Hu F, Liu Y, Meng K, Qian C, Zhang Q, et al: MicroRNA-101a inhibits cardiac fibrosis induced by hypoxia via targeting TGF $\beta$ RI on cardiac fibroblasts. Cell Physiol Biochem 35: 213-226, 2015.

39. Zhang B, Zhou M, Li C, Zhou J, Li H, Zhu D, Wang Z, Chen A and Zhao Q: MicroRNA-92a inhibition attenuates hypoxia/reoxygenation-induced myocardiocyte apoptosis by targeting Smad7. PLoS One 9: e100298, 2014.

40. Zou M, Wang F, Gao R, Wu J, Ou Y, Chen X, Wang T, Zhou X, Zhu W,LiP, et al: Autophagy inhibition of hsa-miR-19a-3p/19b-3p by targeting TGF- $\beta$ R II during TGF- $\beta 1$-induced fibrogenesis in human cardiac fibroblasts. Sci Rep 6: 24747, 2016.

41. Tijsen AJ, van der Made I, van den Hoogenhof MM, Wijnen WJ, van Deel ED, de Groot NE, Alekseev S, Fluiter K, Schroen B, Goumans MJ, et al: The microRNA-15 family inhibits the TGF $\beta$-pathway in the heart. Cardiovasc Res 104: 61-71, 2014

42. Heger J, Warga B, Meyering B, Abdallah Y, Schlüter KD, Piper HM and Euler G: TGF $\beta$ receptor activation enhances cardiac apoptosis via SMAD activation and concomitant NO release. J Cell Physiol 226: 2683-2690, 2011.

43. Ebelt H, Hillebrand I, Arlt S, Zhang Y, Kostin S, Neuhaus H, Müller-Werdan U, Schwarz E, Werdan K and Braun T: Treatment with bone morphogenetic protein 2 limits infarct size after myocardial infarction in mice. Shock 39: 353-360, 2013.

44. Zeisberg M, Hanai J, Sugimoto H, Mammoto T, Charytan D, Strutz F and Kalluri R: BMP-7 counteracts TGF-beta1-induced epithelial-to-mesenchymal transition and reverses chronic renal injury. Nat Med 9: 964-968, 2003.
45. Dobaczewski M, Bujak M, Li N, Gonzalez-Quesada C, Mendoza LH, Wang XF and Frangogiannis NG: Smad3 signaling critically regulates fibroblast phenotype and function in healing myocardial infarction. Circ Res 107: 418-428, 2010.

46. Biernacka A, Cavalera M, Wang J, Russo I, Shinde A, Kong P, Gonzalez-Quesada C, Rai V, Dobaczewski M, Lee DW, et al: Smad3 signaling promotes fibrosis while preserving cardiac and aortic geometry in obese diabetic mice. Circ Heart Fail 8: 788-798, 2015.

47. Tan SM, Zhang Y, Connelly KA, Gilbert RE and Kelly DJ: Targeted inhibition of activin receptor-like kinase 5 signaling attenuates cardiac dysfunction following myocardial infarction. Am J Physiol Heart Circ Physiol 298: H1415-H1425, 2010.

48. Engebretsen KV, Skårdal K, Bjørnstad S, Marstein HS, Skrbic B, Sjaastad I, Christensen G, Bjørnstad JL and Tønnessen T: Attenuated development of cardiac fibrosis in left ventricular pressure overload by SM16, an orally active inhibitor of ALK5. J Mol Cell Cardiol 76: 148-157, 2014.

49. Anderton MJ, Mellor HR, Bell A, Sadler C, Pass M, Powell S, Steele SJ, Roberts RR and Heier A: Induction of heart valve lesions by small-molecule ALK5 inhibitors. Toxicol Pathol 39: 916-924, 2011.

50. Wei LH, Huang XR, Zhang Y, Li YQ, Chen HY, Yan BP, Yu CM and Lan HY: Smad7 inhibits angiotensin II-induced hypertensive cardiac remodelling. Cardiovasc Res 99: 665-673, 2013.

51. Matsumoto-Ida M, Takimoto Y, Aoyama T, Akao M, Takeda T and Kita T: Activation of TGF-beta1-TAK1-p38 MAPK pathway in spared cardiomyocytes is involved in left ventricular remodeling after myocardial infarction in rats. Am J Physiol Heart Circ Physiol 290: H709-H715, 2006. 